\title{
Evaluación de la imagen organizacional universitaria en una institución de educación superior
}

\author{
Evaluation of university organizational image in an institution of \\ higher education
}

\author{
Juana Patlán Pérez ${ }^{\mathrm{a}, *}$ y Edgar Martínez Torres ${ }^{\mathrm{b}}$ \\ a Universidad Nacional Autónoma de México, Ciudad de México, México \\ ${ }^{\mathrm{b}}$ Universidad Autónoma del Estado de Hidalgo, México
}

Recibido el 20 de enero de 2015; aceptado el 28 de enero de 2016

Disponible en Internet el 16 de septiembre de 2016

\begin{abstract}
Resumen
La imagen organizacional es importante para muchas instituciones de educación superior por encontrarse en un entorno ampliamente competitivo que demanda servicios educativos de calidad. El propósito de esta investigación fue evaluar la imagen organizacional universitaria en una institución de educación superior. Para este propósito se realizó una investigación de acuerdo con las siguieron etapas: adaptación de la escala de imagen organizacional a población mexicana; integración de escala en formato de diferencial semántico; aplicación de la escala a una muestra de 226 profesores y 541 estudiantes de la Universidad Autónoma del Estado de Hidalgo; determinación de las propiedades psicométricas de la escala (validez de constructo y confiabilidad); estadísticas descriptivas de la imagen organizacional y análisis comparativo de la imagen organizacional por escuela. Los resultados indican que la escala de imagen organizacional adaptada a población mexicana tiene propiedades psicométricas adecuadas para evaluar este constructo. Además, se identificaron diferencias significativas de la imagen de la organización en cada instituto de la IES evaluada. (C) 2016 Universidad Nacional Autónoma de México, Facultad de Contaduría y Administración. Este es un artículo Open Access bajo la licencia CC BY-NC-ND (http://creativecommons.org/licenses/by-nc-nd/4.0/).
\end{abstract}

Códigos JEL: I23; C38; J24; L14

Palabras clave: Imagen; Imagen organizacional; Imagen organizacional universitaria

\footnotetext{
* Autor para correspondencia.

Correo electrónico: patlanjuana@ hotmail.com (J. Patlán Pérez).

La revisión por pares es responsabilidad de la Universidad Nacional Autónoma de México.
} 


\begin{abstract}
Organizational image is an important issue for many institutions of higher education by being immersed in a competitive environment that requires higher quality of educational services. The objective of this research is to evaluate the organizational image of an institution of higher education. For this purpose, an investigation was conducted. The stages of this research were: translation-retranslation of the original scale of organizational image; integration of scale items using a semantic differential response scale type; piloting of the scale with a sample of 226 teachers and 541 students of Autonomous University of Hidalgo State; determination of the psychometric properties of the scale (construct validity and reliability, and correlations between the factors of scale), descriptive statistics of the scale and comparative analysis. The results indicate that the organizational image scale adapted to Mexican population has adequate psychometric properties to assess this construct. In addition, we identified significant differences of the organizational image in each institute of the IES evaluated.

(C) 2016 Universidad Nacional Autónoma de México, Facultad de Contaduría y Administración. This is an open access article under the CC BY-NC-ND license (http://creativecommons.org/licenses/by-nc-nd/4.0/).
\end{abstract}

JEL classification: I23; C38; J24; L14

Keywords: Image; Organizational image; University organizational image

\title{
Introducción
}

La imagen organizacional es importante para muchas organizaciones que pretenden ser competitivas y garantizar su permanencia en el mercado en el corto, mediano y largo plazo con una demanda creciente por los productos y/o servicios que ofrecen. La imagen percibida por todos los públicos y audiencias de una organización, así como por los empleados tiene repercusiones en el funcionamiento eficaz de las organizaciones (Treadwell y Harrison, 1994; McPherson y Schapiro, 1998; Druteikiene, 2011). En las instituciones de educación superior (IES) la imagen organizacional es un factor importante para atraer y retener a los mejores estudiantes, profesores y empleados (Helgesen y Nesset, 2007; Stevens, McConkey, Cole y Clow, 2008; Polat, 2011). En el caso de los estudiantes la imagen de una institución educativa es importante para realizar sus estudios, lograr la lealtad de los estudiantes y ser un medio para atraer a otros estudiantes (Brown y Mazzarol, 2009). En lo referente a los profesores y el personal, la imagen de la institución educativa es fundamental porque genera vínculos afectivos, produce una respuesta y desempeño positivo, genera mayor compromiso, involucramiento y cohesión en el personal (Treadwell y Harrison, 1994; Herrbach y Mignonac, 2004; Traverso, 2005).

Para la evaluación de la imagen organizacional en la literatura se identifican diversos instrumentos de medición dirigidos a medir, por ejemplo, la imagen de oficiales de policía (Yim y Schafer, 2009), la imagen de colegios (Stevens et al., 2008) y la imagen de universidades e IES de diversos países (Gioia y Thomas, 1996; Magierski y Kassouf, 2003; Baker y Brown, 2007; Helgesen y Nesset, 2007). Además, destacan estudios que miden la imagen organizacional a través de las percepciones de directivos (Herrbach y Mignonac, 2004) y cirujanos (Dukerich, Golden y Shortell, 2002), por citar algunos.

La evaluación de la imagen organizacional universitaria representa un reto toda vez que los instrumentos de medición para IES son escasos, por tal motivo se consideró relevante efectuar la evaluación de la imagen organizacional de una IES a partir de las percepciones de profesores y estudiantes. El objetivo se dirigió a identificar las percepciones que el público interno (profesores 
y estudiantes) de una IES se ha formado a partir de su experiencia, información e interacción con la organización. Para tal propósito se realizó la adaptación de la escala de imagen organizacional universitaria (cognitiva y afectiva) de Beerli, Díaz y Pérez (2002) y Russell y Pratt (1980) a población mexicana. La metodología utilizada en esta investigación consistió en 5 etapas: a) adaptación de la escala original en inglés a población mexicana; b) integración de la escala con un formato de respuesta de tipo diferencial semántico; c) aplicación de la escala a una muestra de 226 profesores y 541 estudiantes de una IES; d) determinación de las propiedades psicométricas de la escala (análisis factorial y análisis de ecuaciones estructurales, análisis de confiabilidad y análisis de correlaciones entre los factores); y e) estadísticas descriptivas y el análisis de la imagen organizacional en las escuelas o institutos de la IES evaluada.

A continuación se analizan los fundamentos conceptuales del constructo imagen organizacional y de imagen organizacional universitaria, así también se analizan los componentes que integran este constructo. Posteriormente se desarrolla el método utilizado y se presentan los resultados y las conclusiones de esta investigación.

\section{Imagen organizacional}

La imagen organizacional es un constructo complejo basado en la percepción del público o personal de una organización que realiza una valoración diferenciadora y comparativa de sus características (Günalan y Ceylan, 2014). La imagen se refiere a la impresión total que una persona construye en su mente acerca de algo o alguien (Dichter, 1985). La imagen se forma en las personas o públicos de la organización como resultado de la interpretación que realizan de la información o desinformación de la organización (Toto y García, 2012). Sin embargo, la imagen involucra las creencias, actitudes, estereotipos, ideas, comportamientos relevantes e impresiones que una persona tiene de un objeto, una persona o una organización (Kotler y Andreasen, 2008).

La imagen es definida como la suma de creencias, ideas e impresiones que una persona tiene de un objeto. Desde el punto de vista práctico la imagen puede ser institucional u organizacional. La imagen institucional se construye a medida que un grupo de personas construyen una institución en cuanto a sus objetivos, su forma de trabajar, el trato que reciben los empleados, que en conjunto se convierte en una imagen institucional que indicará cuál es la ética de la organización. Por su parte, la imagen organizacional se refiere a una imagen natural, espontánea, fruto de las expectativas y el intercambio que tienen las personas con la organización (Giangrande, 1995).

Por su parte Polat (2011) define a la imagen organizacional como la visión, representación o impresión que las personas se forman en su mente en función de la información o datos de una organización obtenidos a través de la interacción con los elementos o componentes de la organización. De esta forma, las organizaciones que logran atraer recursos humanos talentosos o clientes son aquellas que mantienen y comunican una imagen positiva.

La imagen organizacional es el conocimiento compartido que tienen las personas de una organización y de cómo debe operar (Berg, 1985). La imagen organizacional es abordada también como imagen corporativa, prestigio externo percibido, reputación corporativa, identidad corporativa entre otros (Helgesen y Nesset, 2007). No obstante, estos constructos tienen diferencias, tal y como se muestra en la tabla 1.

La imagen organizacional es definida por diversos autores con un enfoque tanto de creencias como de actitudes con respecto a una organización (Kotler, 1975). Sin embargo, Treadwell y Harrison (1994) plantean una perspectiva multifacética de la imagen organizacional concebida como un conjunto de cogniciones, incluyendo creencias, actitudes e impresiones acerca de comportamientos y aspectos relevantes de una organización. En este sentido, la imagen organizacional 
Tabla 1

Definiciones de constructos asociados a la imagen organizacional

\begin{tabular}{|c|c|}
\hline Constructo & Definición \\
\hline Imagen corporativa & $\begin{array}{l}\text { Son las percepciones que tienen diferentes públicos internos y externos de la } \\
\text { organización (Chun, 2005). Involucra las impresiones que tienen personas internas } \\
\text { (empleados, directivos) y externas de la organización (clientes, proveedores). Es un } \\
\text { indicador de la confianza que tiene el cliente con la organización (Huang y Lien, 2012) }\end{array}$ \\
\hline Prestigio externo percibido & $\begin{array}{l}\text { Se refiere a la percepción que tienen las personas externas de la imagen de una } \\
\text { organización. Describe la forma en que las personas externas interpretan y evalúan la } \\
\text { reputación de una organización en función de su experiencia, acercamiento y la } \\
\text { información de que disponen de la organización (Mael y Ashforth, 1992; Herrbach y } \\
\text { Mignonac, 2004). Incluye también las creencias acerca de cómo ven a la organización } \\
\text { las personas externas a ella (Dutton, Dukerich y Harquail, 1994) }\end{array}$ \\
\hline Reputación corporativa & $\begin{array}{l}\text { Es la valoración que realizan diferentes personas (internas y externas) acerca de la } \\
\text { capacidad de la organización para cumplir con sus expectativas en el tiempo (Fombrun } \\
\text { y Van Riel, 2003). En general se refiere a las percepciones públicas de la organización } \\
\text { que son compartidas por múltiples personas en el tiempo }\end{array}$ \\
\hline Identidad corporativa & $\begin{array}{l}\text { Se refiere a la percepción común de lo que la organización hace y cómo funciona, } \\
\text { generando una identificación de las personas (internas y externas) con la organización, } \\
\text { sus metas y sus logros (Gioia y Thomas, 1996). La percepción de la identidad de una } \\
\text { organización es capturada por el constructo de imagen organizacional (Herrbach y } \\
\text { Mignonac, 2004). También es definida como el significado percibido de las } \\
\text { características de la organización (Sartore-Baldwin y Walker, 2011) }\end{array}$ \\
\hline
\end{tabular}

Fuente: integración con base en varios autores.

representa una respuesta subjetiva individual de una persona para diferenciar a la organización con respecto a metáforas, fantasías, mitos o cogniciones más estructuradas como esquemas o mapas mentales. Según añaden Treadwell y Harrison (1994) la imagen organizacional es el resultado, necesariamente, de una comunicación persuasiva o planeada que frecuentemente se realiza en una vía y de forma directa hacia un público o audiencia específica. El proceso social-cognitivo para formar la imagen organizacional implica que cualquier organización es capaz de controlar la memoria de las personas, tanto internas como externas, induciéndoles a olvidar experiencias negativas o incompatibles con su imagen y, en cambio, recordar o resaltar en la mente eventos positivos acordes con la visión y propósitos de la organización.

Para Duque y Carvajal (2015) la imagen organizacional se describe como conocimiento subjetivo, como una actitud y como una combinación de las características del bien o servicio que fabrica una organización. Sin embargo, la imagen organizacional de acuerdo con estos autores forma parte de un conjunto de percepciones individuales acerca de una organización, sus características, procesos y productos (bienes y/o servicios) fabricados o producidos. Así también, la imagen organizacional se identifica por las ideas expresadas por una sociedad que tiene asimetrías y está supeditada a la precepción de los individuos, a los intereses de cada uno y a los roles que desempeña cada persona respecto a una organización determinada (Blázquez y Peretti, 2012).

La imagen organizacional es importante principalmente para el personal de la organización y para el público externo, tal como se muestra en la tabla 2.

\section{Imagen organizacional universitaria}

Nguyen y Leblanc (2001) definen la imagen organizacional universitaria como la imagen percibida que tiene el público de una IES de acuerdo con sus ideas, intereses y experiencias personales, 
Tabla 2

Importancia de la imagen organizacional

\begin{tabular}{lll}
\hline Importancia para: & Autor & Importancia de la imagen organizacional \\
\hline Recursos humanos & Herrbach y Mignonac (2004) & $\begin{array}{l}\text { Las percepciones de los trabajadores contribuyen a un } \\
\text { mejor desempeño } \\
\text { Tiene un efecto significativo en las actitudes y } \\
\text { comportamiento de los empleados: mayor satisfacción }\end{array}$ \\
& & $\begin{array}{l}\text { laboral, compromiso organizacional y bienestar en el trabajo } \\
\text { Permite atraer a candidatos mejor preparados }\end{array}$ \\
& & Retener a empleados con mejor desempeño \\
& Es un factor de atracción de recursos humanos valiosos. \\
& & Es un elemento aglutinador y de conexión entre los \\
Traverso (2005) & miembros de la organización \\
& Es un elemento importante de atracción de públicos \\
externos, mediante atributos instrumentales y significados & simbólicos
\end{tabular}

Fuente: integración basada en varios autores.

sociales e históricas. Bajo esta definición la persona vinculada a la institución universitaria realiza una valoración racional y emocional de los atributos tangibles e intangibles de la institución. De esta forma, cada persona construye mentalmente una imagen de la institución, la cual puede ser distinta en cada persona que evalúe a la institución. Por su parte, Guédez y Osta (2012) consideran que la imagen organizacional universitaria hace referencia a la imagen percibida por su público externo (organizaciones públicas y privadas, egresados, instituciones gubernamentales) e interno (estudiantes actuales, personal docente, personal administrativo y de servicios, entre otros), quienes de acuerdo con su experiencia, intereses e ideas realizan una valoración racional, cognitiva y emocional de las características y atributos de la institución.

En el ámbito de las IES existen diversos estudios acerca de la imagen organizacional de las universidades. Magierski y Kassouf (2003) presentan un análisis de la imagen corporativa de 5 universidades de Sao Pablo mediante la matriz familiaridad-favorabilidad, con lo que fue posible verificar el conocimiento y reputación de las universidades en determinado público, cuyos resultados es posible utilizar para la planeación de marketing de las universidades.

En la literatura ha existido un amplio interés por el estudio de los factores de elección de una institución de educación superior para realizar los estudios universitarios. Entre estos factores destacan el género, la raza, la institución donde realizaron los estudios de bachillerato, la clase social, la influencia de familiares, la influencia de compañeros y profesores y la imagen y reputación de la institución educativa (Baker y Brown, 2007).

La imagen organizacional universitaria es importante para el funcionamiento de una IES, para los estudiantes y para el personal de este tipo de organizaciones. A manera de resumen, en la tabla 3 se presentan los factores de importancia de la imagen organizacional universitaria para IES.

Es importante añadir que las IES deben preocuparse por la imagen organizacional universitaria por 3 razones principales (Torpor, 1983): a) este tipo de instituciones necesitan saber cómo se percibe la institución con respecto a la competencia; b) es necesario conocer cómo son percibidas estas instituciones por sus diversas audiencias (estudiantes, exalumnos, sociedad); y c) se requiere monitorizar los cambios que se tengan en la imagen de la institución y, con ello, estar en posibilidad de reducir la brecha entre imagen real y la imagen deseada o esperada. En consecuencia, la evaluación de la imagen organizacional universitaria es una tarea importante. 
Tabla 3

Importancia de la imagen organizacional universitaria

\begin{tabular}{|c|c|c|}
\hline Importancia para & Autor & $\begin{array}{l}\text { Importancia de la imagen organizacional } \\
\text { universitaria }\end{array}$ \\
\hline \multirow[t]{5}{*}{$\begin{array}{l}\text { Funcionamiento y } \\
\text { competitividad de la } \\
\text { organización universitaria }\end{array}$} & Treadwell y Harrison (1994) & $\begin{array}{l}\text { Contribuye al buen funcionamiento de la } \\
\text { organización y genera mejores resultados de la } \\
\text { institución educativa }\end{array}$ \\
\hline & McPherson y Schapiro (1998) & $\begin{array}{l}\text { Contribuye a lograr la competitividad de la } \\
\text { institución educativa, distinguiéndola de sus } \\
\text { competidores, mejorando el prestigio y la calidad } \\
\text { educativa }\end{array}$ \\
\hline & Druteikiene (2011) & La imagen es una fuente de ventaja competitiva \\
\hline & Blázquez y Peretti (2012) & $\begin{array}{l}\text { Tiene como resultado la sustentabilidad de la } \\
\text { organización }\end{array}$ \\
\hline & Helgesen y Nesset (2007) & Contribuye a lograr la lealtad de los estudiantes \\
\hline \multirow[t]{2}{*}{ Estudiantes } & Stevens et al., 2008 & $\begin{array}{l}\text { Genera resultados positivos para que los estudiantes } \\
\text { recomienden a la institución educativa }\end{array}$ \\
\hline & Polat (2011) & $\begin{array}{l}\text { Logra la satisfacción de los estudiantes mediante } \\
\text { profesores destacados, una buena infraestructura, } \\
\text { recursos y servicios adecuados }\end{array}$ \\
\hline \multirow[t]{3}{*}{ Recursos humanos } & Treadwell y Harrison (1994) & $\begin{array}{l}\text { Genera vínculos afectivos entre los trabajadores y la } \\
\text { organización }\end{array}$ \\
\hline & & $\begin{array}{l}\text { Produce una respuesta positiva en los trabajadores, } \\
\text { generando mayor compromiso e involucramiento } \\
\text { del personal }\end{array}$ \\
\hline & Nolan y Harold (2010) & $\begin{array}{l}\text { Atrae y retiene al recurso humano a las } \\
\text { organizaciones }\end{array}$ \\
\hline
\end{tabular}

Fuente: Integración basada en varios autores.

\section{Componentes de la imagen organizacional universitaria}

Nguyen y Leblanc (2001) señalan la presencia de 2 componentes de la imagen organizacional: funcional y emocional. El componente funcional está relacionado con las características tangibles de la organización que pueden ser fácilmente medidas, en cambio el componente emocional se relaciona con aspectos psicológicos tales como sentimientos y actitudes hacia la organización como resultado de las experiencias y el procesamiento de información de atributos que contribuyen al funcionamiento de la organización.

Galiniené, Marcinskas, Miskinis y Druteikiene (2009) identifican diferentes atributos de la imagen organizacional, por ejemplo la ubicación geográfica, el tipo de universidad (pública o privada), la complejidad para el ingreso y el nivel educativo de los aspirantes, los premios y reconocimientos obtenidos por los profesores, la calidad de los estudios, los programas ofrecidos, el acervo bibliográfico, el presupuesto de la institución, las cuotas o monto de matrícula, etc. De acuerdo con estos autores la imagen ideal de una IES considera 4 factores (cognición, evaluación, actividad y fortalezas) y 11 componentes: confiable, cálida, activa, amigable, liberal, reputación, atractiva, en desarrollo, joven, moderna y abierta.

En general, las definiciones de imagen organizacional contemplan un componente cognitivo basado en el conjunto de creencias o atributos percibidos de la organización. Sin embargo, existe una dimensión que captura sentimientos acerca del objeto evaluado. Beerli et al., 2002 sustentan que la imagen organizacional de una universidad es un fenómeno perceptual que está formado por una valoración e interpretación racional y emocional que hace la persona de la organización y, 
Tabla 4

Componentes de la imagen organizacional universitaria

\begin{tabular}{lll}
\hline Autores & Núm. & Componentes de la imagen organizacional universitaria \\
\hline Nguyen y Leblanc (2001) & 2 & $\begin{array}{l}\text { Componente funcional } \\
\text { Componente emocional } \\
\text { Imagen cognitiva (creencias) }\end{array}$ \\
Galiniené et al., 2009 & 2 & $\begin{array}{l}\text { Imagen afectiva (sentimientos, emociones) } \\
\text { Imagen cognitiva }\end{array}$ \\
& 3 & $\begin{array}{l}\text { Imagen emocional-afectiva } \\
\text { Imagen general }\end{array}$ \\
Guerra y Arends (2008) y Traverso (2005) & 6 & $\begin{array}{l}\text { Calidad académica } \\
\text { Aspectos sociales }\end{array}$ \\
& & $\begin{array}{l}\text { Elementos tangibles } \\
\text { Calidad del servicio }\end{array}$ \\
Accesibilidad \\
Aspectos laborales \\
Atributos instrumentales \\
Significados simbólicos \\
Accesibilidad \\
Calidad de servicio \\
Calidad académica \\
Elementos tangibles \\
\end{tabular}

Fuente: integración basada en varios autores.

por tanto, se integra de un sistema indisociable de componentes: cognitivo (creencias) y afectivo (sentimientos, emociones).

Por su parte, Galiniené et al., 2009 señalan que la imagen organizacional universitaria se integra de 3 componentes:

- Imagen cognitiva. Se refiere a las cogniciones desarrolladas con respecto a las instalaciones, los cursos, el clima, las oportunidades de admisión, el costo de la matrícula, los docentes, la calidad de la educación, la preparación de los estudiantes, el enfoque teórico-práctico, los requisitos de admisión, la orientación o enfoque hacia los estudiantes, la comunicación o distancia entre el alumno y el profesor, la relación o distancia entre la universidad y la sociedad, la vinculación de la universidad con las empresas, el número de alumnos, la popularidad de la universidad, la edad de la universidad y el tipo de universidad (de élite, tradicional, innovadora).

- Imagen emocional-afectiva. Involucra emociones tales como agradable-desagradable, aburridoestimulante, estresante-relajado, sombrío-animado.

- Imagen general. Se refiere a la percepción positiva o negativa que se tiene de una organización.

De acuerdo con los antecedentes analizados previamente, se identifica que el constructo de imagen organización está integrado por un componente cognitivo y otro afectivo, sin dejar de lado la presencia de indicadores de tipo global (tabla 4).

Por tanto, el objetivo de esta investigación fue evaluar la imagen organizacional en una IES mediante la adaptación a población mexicana de la escala de imagen cognitiva de Beerli et al., 2002 y la escala de imagen afectiva de Russell y Pratt (1980) para IES. Adicionalmente, en esta investigación se identificó la imagen organizacional universitaria percibida por escuela. 


\section{Método}

\section{Planteamiento y pregunta de investigación}

La imagen organizacional es relevante para las organizaciones y, particularmente, para las IES. La demanda por cursar estudios universitarios se ve influida por la imagen universitaria, así también la atracción y retención de profesores y empleados se ve afectada por la imagen que tiene la institución. También la imagen organizacional es importante porque es un elemento básico en la dirección estratégica, es un factor de atracción hacia las organizaciones y es un factor aglutinador y de cohesión de los miembros de la organización (Traverso, 2005).

Según lo señalado por Perozo y Alcalá (2008) la imagen es importante tanto para la fuente de la imagen (organización) como para quien la recibe (el sujeto). Para la organización una imagen positiva es requisito para establecer una relación positiva-favorable con un público meta, en cambio para el sujeto la imagen es una forma de considerar o valorar a la organización (buena, mala, útil, inútil, etc.). De esta forma cuanto mayor es la confianza que el sujeto pone en la imagen de la organización, más importante será que la organización tenga una reputación sólida. En este sentido se consideró relevante realizar una investigación con el propósito de conocer cuál es la imagen organizacional percibida en una la Universidad Autónoma del Estado de Hidalgo, tanto por sus profesores como por sus estudiantes, así como identificar las diferencias significativas que existen en la imagen percibida en cada instituto de la IES evaluada.

\section{Tipo y diseño de investigación}

Se realizó una investigación ex-post facto, de tipo descriptiva y de asociación de variables. Se utilizó un diseño no experimental.

\section{Muestra}

La muestra se integró de 541 (70.5\%) estudiantes de licenciatura y 226 (29.5\%) profesores de tiempo completo de una IES (tabla 1). La edad promedio de la muestra fue de 27.2 años, $51.0 \%$ son mujeres y $49.0 \%$ hombres. El $24.5 \%$ de la muestra corresponde al Instituto de Ciencias Económico Administrativas, 20.0\% al Instituto de Ciencias Básicas e Ingeniería, 19.3\% al Instituto de Ciencias de la Salud, $15.9 \%$ al Instituto de Ciencias Sociales y Humanidades, $11.5 \%$ al Instituto de Artes y el $8.2 \%$ al Instituto de Ciencias Agropecuarias.

\section{Instrumento}

Para la medición de la imagen cognitiva se utilizaron 21 reactivos en escala de diferencial semántico compuesta por adjetivos bipolares con 5 puntos de respuesta $(5,4,3,2,1)$ la cual fue desarrollada por Beerli et al., 2002 para IES. También se utilizó el indicador global de imagen organizacional construido por estos autores, correspondiente a un reactivo. Para la medición de la imagen afectiva se utilizaron 3 reactivos desarrollados por Russell y Pratt (1980) para IES y validados previamente por Beerli et al., 2002. El instrumento de medición y los reactivos finales de la escala se presentan en el anexo 1. 


\section{Procedimiento}

La metodología utilizada consistió en 7 etapas: a) adaptación de la escala original en inglés de Beerli et al., 2002 y Russell y Pratt (1980) mediante un proceso de traducción-retraducción efectuado por 2 expertos en evaluación organizacional y psicometría; b) integración de la escala y los reactivos, en este caso los reactivos fueron comprensibles y congruentes para la población mexicana y el formato de respuesta fue de tipo diferencial semántico, con 5 puntos de respuesta entre los pares de adjetivos de la escala; c) aplicación de la escala a una muestra de 226 profesores y 541 estudiantes de una IES; d) determinación de las propiedades psicométricas de la escala (análisis factorial y análisis de ecuaciones estructurales, análisis de confiabilidad y análisis de correlaciones entre factores); y e) estadísticas descriptivas de la imagen organizacional y análisis comparativos por escuela.

\section{Análisis de datos}

La validez de constructo se realizó mediante el análisis factorial utilizando el software SPSS versión 17 y el análisis de ecuaciones estructurales con el software AMOS versión 20. Para determinar la confiabilidad de la escala se estimó el alfa de Cronbach, además se realizaron estadísticas descriptivas a los factores de la escala y se determinaron los coeficientes de correlación de Pearson entre los factores de la escala. Para identificar las relaciones estadísticamente significativas entre los factores de la imagen organizacional y los diferentes institutos de la IES evaluada se realizó el análisis de la varianza de una vía.

\section{Resultados y discusión}

El proceso de adaptación de la escala de imagen organizacional de Beerli et al., 2002 y Russell y Pratt (1980) se efectuó mediante un proceso de traducción y retraducción de los reactivos de la escala original en inglés con la colaboración de 2 expertos en evaluación organizacional y psicometría. En este proceso se conservó el significado original del reactivo. Posteriormente se integraron los reactivos de la escala de forma tal que estos fueran comprensibles y congruentes para población mexicana. Se integró un total de 23 reactivos (19 reactivos para imagen cognitiva, 3 reactivos para imagen afectiva, un reactivo para el indicador global), los cuales contaron con una escala de respuesta de tipo diferencial semántico con 5 puntos de respuesta entre los pares de adjetivos bipolares de la escala. La aplicación de la escala se efectuó con una muestra de 226 profesores y 541 estudiantes de una IES de México.

En la tabla 5 se presentan los resultados de la validez de constructo de la Escala de imagen organizacional universitaria efectuada mediante el análisis factorial con el método de componentes principales y rotación varimax. Los resultados arrojan la presencia de 3 factores de imagen cognitiva (F1 orientación y preparación universitaria, F2 reputación de la institución, F3 madurez institucional) y un factor de imagen afectiva (F4). El porcentaje de varianza explicada acumulada fue de $70.35 \%$, con un porcentaje de varianza explicada de $21.97 \%$ para el primer factor, $18.85 \%$ para el segundo, $14.86 \%$ para el tercero y $16.67 \%$ para el cuarto. En estos resultados se obtuvo un valor de 0.889 en la medida de adecuación muestral de Kaiser-Meyer-Olkin y de 5929.49 en la prueba de esfericidad de Bartlet $(\mathrm{p}=0.000)$, lo que indica la idoneidad del análisis factorial. En total se eliminaron 7 reactivos en los factores de imagen cognitiva y un reactivo en el factor de imagen afectiva. 
Tabla 5

Análisis factorial de la Escala de imagen organizacional universitaria

\begin{tabular}{|c|c|c|c|c|}
\hline Reactivos & $\begin{array}{l}\text { F1 Imagen cognitiva: } \\
\text { orientación y } \\
\text { preparación } \\
\text { universitaria }\end{array}$ & $\begin{array}{l}\text { F2 Imagen cognitiva: } \\
\text { reputación de la } \\
\text { institución }\end{array}$ & $\begin{array}{l}\text { F3 Imagen } \\
\text { cognitiva: } \\
\text { madurez } \\
\text { institucional }\end{array}$ & $\begin{array}{l}\text { F4 Imagen } \\
\text { afectiva }\end{array}$ \\
\hline $\begin{array}{l}\text { 1. La enseñanza de los profesores es } \\
\text { buena/mala }\end{array}$ & 0.675 & 0.082 & 0.072 & 0.507 \\
\hline 2. La educación es buena/mala & 0.675 & 0.142 & 0.061 & 0.486 \\
\hline $\begin{array}{l}\text { 3. Los profesores son muy } \\
\text { exigentes/no son muy exigentes }\end{array}$ & 0.699 & 0.096 & 0.152 & 0.011 \\
\hline $\begin{array}{l}\text { 4. Centrada en el estudiante/no } \\
\text { centrada en el estudiante }\end{array}$ & 0.775 & 0.268 & 0.197 & 0.074 \\
\hline $\begin{array}{l}\text { 5. Cercana a los } \\
\text { estudiantes/distanciada de los } \\
\text { estudiantes }\end{array}$ & 0.770 & 0.234 & 0.187 & 0.117 \\
\hline $\begin{array}{l}\text { 6. Universidad } \\
\text { innovadora/tradicional }\end{array}$ & 0.432 & 0.448 & 0.343 & 0.183 \\
\hline $\begin{array}{l}\text { 7. Universidad actualizada/no } \\
\text { actualizada }\end{array}$ & 0.264 & 0.658 & 0.343 & 0.152 \\
\hline $\begin{array}{l}\text { 8. Cuenta con un buen prestigio/mal } \\
\text { prestigio }\end{array}$ & 0.179 & 0.872 & 0.159 & 0.192 \\
\hline $\begin{array}{l}\text { 9. Tiene una buena reputación/mala } \\
\text { reputación }\end{array}$ & 0.178 & 0.840 & 0.102 & 0.290 \\
\hline $\begin{array}{l}\text { 10. Cuenta con buenas instalaciones/ } \\
\text { malas instalaciones }\end{array}$ & 0.271 & 0.128 & 0.749 & 0.184 \\
\hline $\begin{array}{l}\text { 11. Con gran variedad de programas } \\
\text { educativos/con pocos programas } \\
\text { educativos }\end{array}$ & 0.109 & 0.270 & 0.777 & 0.067 \\
\hline 12. El ambiente es bueno/malo & 0.160 & 0.124 & 0.633 & 0.502 \\
\hline 13. Institución estimulante/aburrida & 0.182 & 0.433 & 0.255 & 0.716 \\
\hline 14. Institución relajante/estresante & 0.137 & 0.291 & 0.215 & 0.767 \\
\hline Porcentaje de varianza explicada & 21.97 & 18.85 & 14.86 & 16.67 \\
\hline $\begin{array}{l}\text { Porcentaje de varianza explicada } \\
\text { acumulada }\end{array}$ & 21.97 & 40.82 & 55.69 & 70.35 \\
\hline $\begin{array}{l}\text { Medida de adecuación muestral de } \\
\text { Kaiser-Meyer-Olkin }\end{array}$ & 0.889 & & & \\
\hline Prueba de esfericidad de Bartlett & 5929.49 & & & \\
\hline $\mathrm{p}$ & 0.000 & & & \\
\hline
\end{tabular}

Método: componentes principales; rotación: Varimax; $n=767$.

Fuente: elaboración propia basada en los resultados de la investigación.

Así también, se identificó la estructura factorial de la escala de imagen organizacional universitaria mediante el análisis de ecuaciones estructurales. Para este propósito se diseñaron 2 modelos. El modelo 1 incluye 3 factores de imagen cognitiva (F1 orientación y preparación universitaria, F2 reputación de la institución, F3 madurez institucional). Por su parte, el modelo 2 incluyó 3 factores de imagen cognitiva (F1 orientación y preparación universitaria, F2 reputación de la institución, F3 madurez institucional) y un factor de imagen afectiva (F4 imagen afectiva). Los resultados del modelo 1 se presentan en la figura 1. Los valores beta estandarizados de cada reactivo con sus factores respectivos oscilaron entre 0.560 y 0.930 . Además, las correlaciones entre los 3 factores de imagen cognitiva oscilaron entre 0.583 y 0.661 . 


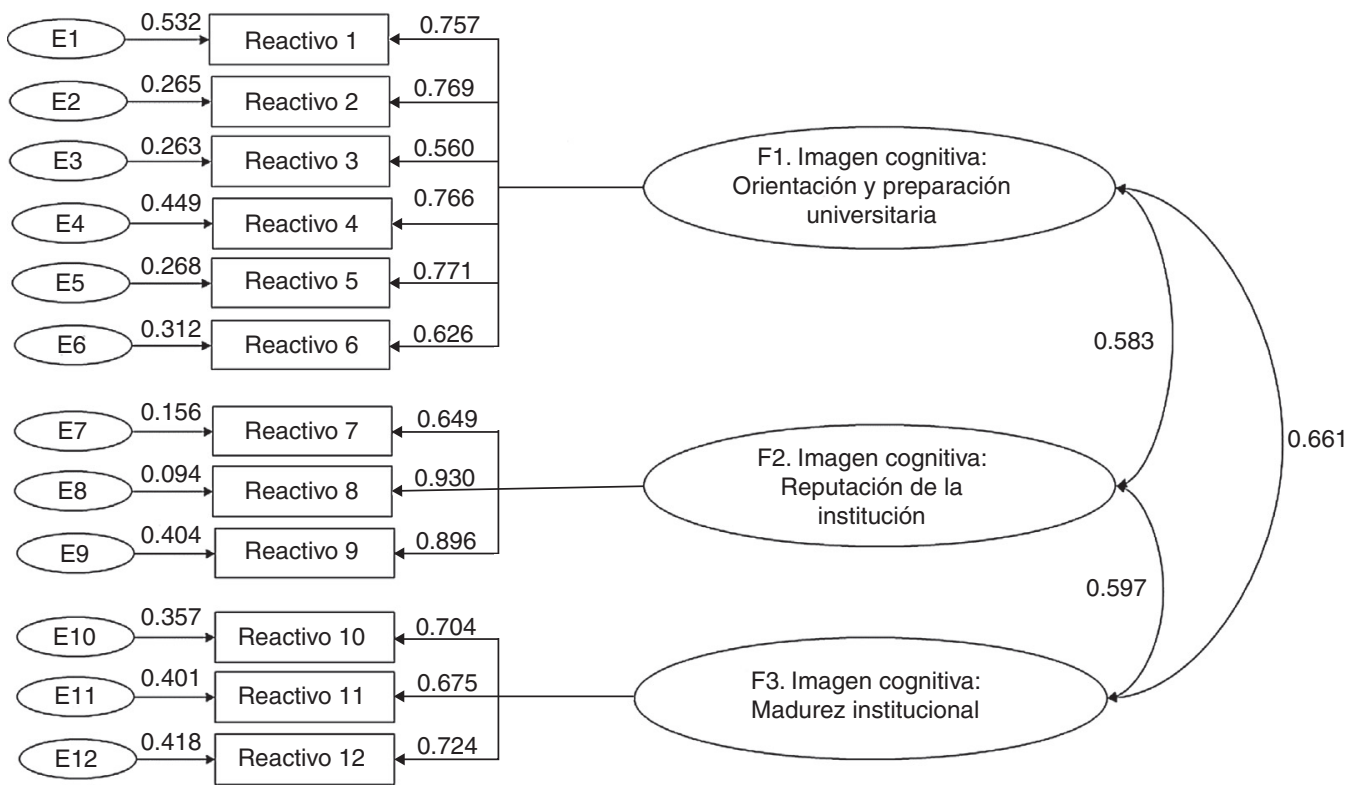

Figura 1. Análisis factorial de la imagen organizacional universitaria: modelo 1.

Fuente: elaboración propia en función de los resultados de la investigación.

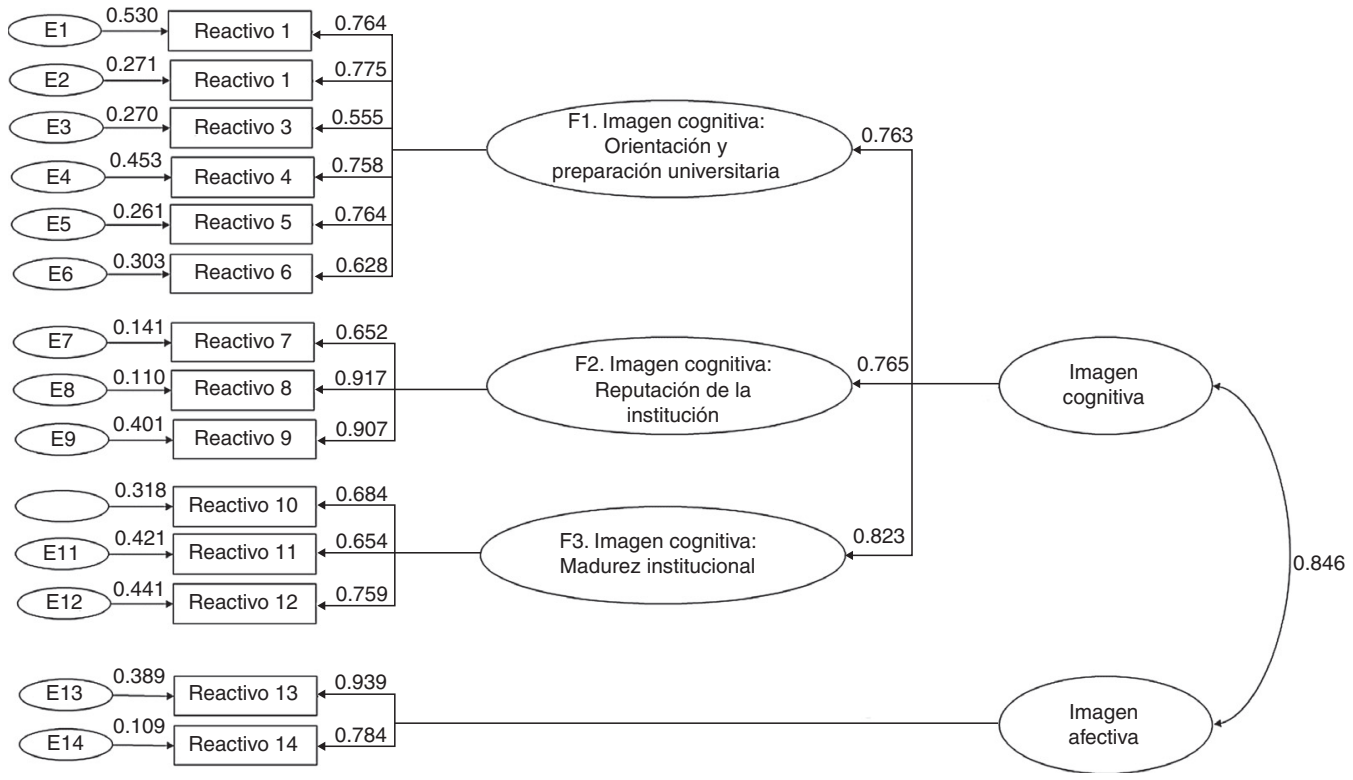

Figura 2. Análisis factorial de la imagen organizacional universitaria: modelo 2.

Fuente: elaboración propia en función de los resultados de la investigación. 
Tabla 6

Medidas de ajuste de los modelos del análisis factorial de la Escala de imagen organizacional universitaria

\begin{tabular}{lcc}
\hline Medidas de ajuste & $\begin{array}{c}\text { Modelo 1. Imagen } \\
\text { cognitiva: 3 factores }\end{array}$ & $\begin{array}{c}\text { Modelo 2. Imagen cognitiva: 3 } \\
\text { factores; Imagen afectiva: un factor }\end{array}$ \\
\hline $\begin{array}{l}\text { Medidas absolutas de ajuste } \\
\mathrm{X}^{2}\end{array}$ & 69.606 & 703.044 \\
$\mathrm{gl}$ & 51 & 73 \\
$\mathrm{p}$ & 0.000 & 0.000 \\
GFI & 0.882 & 0.893 \\
$\mathrm{RMSEA}$ & 0.120 & 0.106 \\
ECVI & 0.898 & 1.038 \\
Medidas de ajuste incremental & \\
TLI & 0.846 & 0.866 \\
NFI & 0.872 & 0.882 \\
CFI & 0.881 & 0.893 \\
Medidas de ajuste parsimonioso & \\
PNFI & 0.674 & 0.708 \\
PGFI & 0.681 & 0.716 \\
CMIN/GL & 11.953 & 703.044 \\
AIC & 687.606 & 795.044 \\
\hline n & & \\
Fuente: elaboración propia basada en los resultados de la investigación.
\end{tabular}

En la figura 2 se presentan los resultados del modelo 2. En estos resultados se identificaron 3 factores de imagen cognitiva y un factor de imagen afectiva. La relación de los factores 1,2 y 3 con la imagen cognitiva osciló entre 0.763 y 0.823 . La correlación entre la imagen cognitiva y la imagen afectiva arrojó un valor de 0.846 . Por lo que se refiere a los valores beta estandarizados de los reactivos con cada factor oscilaron entre 0.555 y 0.939 .

En la tabla 6 se presentan los resultados de las medidas de ajuste, de ajuste incremental y de ajuste parsimonioso de los modelos 1 y 2 cuyos resultados se presentaron previamente. El modelo 2 , que incluye 3 factores de imagen cognitiva y un factor de imagen afectiva arroja mejores niveles de ajuste $\left(\mathrm{X}^{2}=703.044 ; g l=73 ; \mathrm{p}=0.000 ; \mathrm{GFI}=0.893\right.$; RMSEA $\left.=0.106\right)$, comparativamente con el modelo 1. Estos resultados confirman la presencia de 4 factores en la escala de imagen organizacional: 3 factores correspondientes a la imagen cognitiva (orientación y preparación universitaria, reputación de la institución y madurez institucional) y un factor correspondiente a la imagen afectiva.

Las estadísticas descriptivas de los reactivos que conformaron la Escala de imagen organizacional universitaria se presentan en la tabla 7. Todos los reactivos mostraron correlaciones estadísticamente significativas con su factor respectivo y con el total de la escala, con correlaciones significativas $(\mathrm{p} \leq 0.01)$ que oscilaron entre $\mathrm{r}=0.528 \mathrm{y} \mathrm{r}=0.772$.

Los resultados del análisis de confiabilidad arrojaron valores de alfa de Cronbach superiores a 0.70 (tabla 8). En los 3 factores de imagen cognitiva estos indicadores son superiores a 0.80 y en el factor de imagen afectiva el alfa de Cronbach fue de $\alpha=0.744$. La escala global que incluye los 14 reactivos validados presentó un alfa de Cronbach de $\alpha=0.910$.

En la tabla 9 se presentan los resultados descriptivos de la imagen organizacional. El puntaje promedio más alto se identificó en el F2 reputación de la institución (media $=3.59)$ y el puntaje más bajo en el indicador global de la imagen organizacional (media =3.19). 
Tabla 7

Estadísticas descriptivas y correlaciones de los reactivos de la Escala de imagen organizacional universitaria

\begin{tabular}{|c|c|c|c|c|c|c|}
\hline Reactivos & Media & Mediana & $\mathrm{DE}$ & $\begin{array}{l}\text { Correlación } \\
\text { con el factor }\end{array}$ & $\begin{array}{l}\text { Correlación } \\
\text { con el total }\end{array}$ & $\begin{array}{l}\text { Alfa si se elimina } \\
\text { el reactivo }\end{array}$ \\
\hline \multicolumn{7}{|c|}{ F1. Imagen cognitiva: orientación y preparación universitaria } \\
\hline Reactivo 1 & 3.52 & 4.00 & 0.86 & $0.790 *$ & $0.685^{*}$ & 0.821 \\
\hline Reactivo 2 & 3.56 & 4.00 & 0.81 & $0.797 *$ & $0.700 *$ & 0.819 \\
\hline Reactivo 3 & 3.39 & 3.00 & 0.81 & $0.667 *$ & $0.528 *$ & 0.850 \\
\hline Reactivo 4 & 3.40 & 3.00 & 0.79 & $0.805^{*}$ & $0.695 *$ & 0.816 \\
\hline Reactivo 5 & 3.40 & 3.00 & 0.81 & $0.806^{*}$ & $0.689 *$ & 0.816 \\
\hline Reactivo 6 & 3.32 & 3.00 & 0.94 & $0.707 *$ & $0.715^{*}$ & 0.849 \\
\hline \multicolumn{7}{|c|}{ F2. Imagen cognitiva: reputación de la institución } \\
\hline Reactivo 7 & 3.47 & 4.00 & 0.84 & $0.809 *$ & $0.709 *$ & 0.912 \\
\hline Reactivo 8 & 3.66 & 4.00 & 0.83 & $0.923 *$ & $0.711 *$ & 0.708 \\
\hline Reactivo 9 & 3.63 & 4.00 & 0.89 & $0.909 *$ & $0.716^{*}$ & 0.749 \\
\hline \multicolumn{7}{|c|}{ F3. Imagen cognitiva: madurez institucional } \\
\hline Reactivo 10 & 3.33 & 3.00 & 0.91 & $0.820 *$ & $0.642 *$ & 0.661 \\
\hline Reactivo 11 & 3.64 & 4.00 & 0.86 & $0.805^{*}$ & $0.583 *$ & 0.667 \\
\hline Reactivo 12 & 3.49 & 4.00 & 0.87 & $0.814 *$ & $0.668 *$ & 0.650 \\
\hline \multicolumn{7}{|c|}{ F4. Imagen afectiva } \\
\hline Reactivo 13 & 3.42 & 3.00 & 0.96 & $0.928 *$ & $0.772 *$ & - \\
\hline Reactivo 14 & 3.24 & 3.00 & 1.00 & $0.935^{*}$ & $0.685^{*}$ & - \\
\hline \multicolumn{7}{|c|}{ Indicador global de imagen organizacional universitaria } \\
\hline Reactivo 15 & 3.47 & 3.00 & 0.95 & & $0.690 *$ & - \\
\hline
\end{tabular}

$\mathrm{n}=767 ; * \mathrm{p} \leq 0.01$.

Fuente: elaboración propia basada en los resultados de la investigación.

Tabla 8

Coeficientes de confiabilidad de la Escala de imagen organizacional universitaria

\begin{tabular}{llc}
\hline Factor & Alfa de Cronbach & Número de reactivos \\
\hline F1. Imagen cognitiva: orientación y preparación universitaria & 0.853 & 6 \\
F2. Imagen cognitiva: reputación de la institución & 0.855 & 3 \\
F3. Imagen cognitiva: madurez institucional & 0.847 & 3 \\
F4. Imagen afectiva & 0.744 & 2 \\
Global (14 reactivos) & 0.910 & 14
\end{tabular}

$\mathrm{n}=767$

Fuente: elaboración propia basada en los resultados de la investigación.

Tabla 9

Estadísticas descriptivas de la Escala de imagen organizacional universitaria

\begin{tabular}{|c|c|c|c|c|c|c|}
\hline Factor & Media & Mediana & Moda & $\mathrm{DE}$ & Mínimo & Máximo \\
\hline F1. Imagen cognitiva: orientación y preparación universitaria & 3.43 & 3.33 & 3.00 & 0.64 & 1.00 & 5.00 \\
\hline F2. Imagen cognitiva: reputación de la institución & 3.59 & 3.67 & 4.00 & 0.75 & 1.00 & 5.00 \\
\hline F3. Imagen cognitiva: madurez institucional & 3.49 & 3.66 & 4.00 & 0.72 & 1.00 & 5.00 \\
\hline F4 Imagen afectiva. & 3.33 & 3.50 & 3.00 & 0.91 & 1.00 & 5.00 \\
\hline Indicador global de imagen organizacional universitaria & 3.46 & 3.00 & 3.00 & 0.59 & 1.00 & 5.00 \\
\hline
\end{tabular}

$\mathrm{n}=767$.

Fuente: elaboración propia con base en los resultados de la investigación. 
Tabla 10

Coeficientes de correlación de Pearson entre los factores de la Escala de imagen organizacional universitaria

\begin{tabular}{|c|c|c|c|c|c|}
\hline $\begin{array}{l}\text { Factores de la escala de imagen organizacional } \\
\text { universitaria }\end{array}$ & $\mathrm{F} 1$ & $\mathrm{~F} 2$ & $\mathrm{~F} 3$ & $\mathrm{~F} 4$ & Indicador global \\
\hline $\begin{array}{l}\text { F1. Imagen cognitiva: orientación y preparación } \\
\text { universitaria }\end{array}$ & 1 & & & & \\
\hline F2. Imagen cognitiva: reputación de la institución & $0.575^{*}$ & 1 & & & \\
\hline F3. Imagen cognitiva: madurez institucional & $0.543^{*}$ & $0.534 *$ & 1 & & \\
\hline F4. Imagen afectiva & $0.554 *$ & $0.597 *$ & $0.548 *$ & 1 & \\
\hline Indicador global de imagen organizacional universitaria & $0.512 *$ & $0.579 *$ & $0.531 *$ & $0.719 *$ & 1 \\
\hline
\end{tabular}

$\mathrm{n}=767$.

$* \mathrm{p} \leq 0.01$.

Fuente: elaboración propia basada en los resultados de la investigación.

Tabla 11

Definición de factores de la Escala de imagen organizacional universitaria

\begin{tabular}{lll}
\hline Variable Factores &
\end{tabular}

Imagen cognitiva

Conjunto de cogniciones desarrolladas por una persona interna o externa a la organización con respecto a la orientación y preparación universitaria de la IES, su reputación y su madurez institucional

Imagen afectiva

Conjunto de emociones positivas o negativas expresadas por miembros internos y externos de una organización
F1. Orientación y preparación universitaria. Imagen percibida con respecto a atributos relacionados con la orientación de la universidad hacia los estudiantes, la sociedad, así como con la preparación que provee a los estudiantes

F2. Reputación de la institución. Imagen percibida del prestigio y reputación de la institución educativa y su nivel de actualización

F3. Madurez institucional. Imagen percibida del grado de madurez de la institución con respecto a sus instalaciones, programas educativos y ambiente F4. Imagen afectiva. Conjunto de emociones expresadas con respecto a una IES: estimulante-aburrida y relajante-estresante

Fuente: elaboración propia basada en los resultados de la investigación.

Adicionalmente, se identificaron correlaciones significativas entre los 4 factores de la Escala de imagen organizacional que oscilan entre $r=0.512$ y $r=0.597(p \leq 0.01)$. Con el indicador global de imagen organizacional el F4 imagen afectiva obtuvo la correlación significativa más alta $(\mathrm{r}=0.719 ; \mathrm{p} \leq 0.01)$ (tabla 10$)$.

Una vez obtenida la estructura factorial y la confiabilidad de la Escala de imagen organizacional universitaria los factores quedaron definidos tal como se señala en la tabla 11.

Con los resultados previamente señalados, se obtuvo un total de 14 reactivos que miden 4 factores de imagen organizacional universitaria ( 3 factores de imagen cognitiva y un factor de imagen afectiva). La Escala de imagen organizacional universitaria se presenta en el anexo 1.

Por lo que se refiere al análisis comparativo de la imagen organizacional percibida en cada uno de los institutos de la IES evaluada, se identificaron diferencias significativas en todos los factores considerados de imagen organizacional, tomando en cuenta los 6 institutos que integran la IES evaluada.

En la tabla 12 se presentan los resultados del análisis de la varianza. Para identificar las diferencias entre los grupos de medias se efectuó la prueba de Tukey, los resultados obtenidos en esta prueba se reportan en el tabla 12. Estos resultados nos indican que la imagen respecto a 
Tabla 12

Imagen organizacional por escuela (resultados del análisis de la varianza de una vía)

\begin{tabular}{|c|c|c|c|c|c|c|c|}
\hline Factor & Instituto & $\mathrm{n}$ & Media & $\mathrm{DE}$ & $\mathrm{F}$ & $\mathrm{p}$ & Prueba de Tukey \\
\hline \multirow{7}{*}{$\begin{array}{l}\text { F1. Imagen cognitiva: } \\
\text { orientación y preparación } \\
\text { universitaria }\end{array}$} & 1. CEA & 188 & 3.33 & 0.65 & \multirow[t]{7}{*}{2.195} & \multirow[t]{7}{*}{0.050} & \multirow[t]{7}{*}{$1 / 5$} \\
\hline & 2. A & 88 & 3.34 & 0.57 & & & \\
\hline & 3. CBI & 158 & 3.46 & 0.57 & & & \\
\hline & 4. CSHU & 122 & 3.48 & 0.71 & & & \\
\hline & 5. CSA & 148 & 3.53 & 0.68 & & & \\
\hline & 6. CAP & 63 & 3.45 & 0.56 & & & \\
\hline & Total & 767 & 3.43 & 0.64 & & & \\
\hline \multirow{7}{*}{$\begin{array}{l}\text { F2. Imagen cognitiva: } \\
\text { reputación de la } \\
\text { institución }\end{array}$} & 1. CEA & 188 & 3.62 & 0.70 & \multirow[t]{7}{*}{5.119} & \multirow[t]{7}{*}{0.000} & \multirow[t]{7}{*}{$2, / 1,3,4,5$} \\
\hline & 2. A & 88 & 3.25 & 0.82 & & & \\
\hline & 3. CBI & 158 & 3.68 & 0.66 & & & \\
\hline & 4. CSHU & 122 & 3.62 & 0.83 & & & \\
\hline & 5. CSA & 148 & 3.67 & 0.73 & & & \\
\hline & 6. CAP & 63 & 3.46 & 0.81 & & & \\
\hline & Total & 767 & 3.59 & 0.75 & & & \\
\hline \multirow{7}{*}{$\begin{array}{l}\text { F3. Imagen cognitiva: } \\
\text { madurez institucional }\end{array}$} & 1. CEA & 188 & 3.56 & 0.68 & \multirow[t]{7}{*}{6.843} & \multirow[t]{7}{*}{0.000} & \multirow[t]{7}{*}{$6 / 3$} \\
\hline & 2. A & 88 & 3.36 & 0.60 & & & \\
\hline & 3. CBI & 158 & 3.64 & 0.63 & & & \\
\hline & 4. CSHU & 122 & 3.45 & 0.73 & & & \\
\hline & 5. CSA & 148 & 3.51 & 0.73 & & & \\
\hline & 6. CAP & 63 & 3.08 & 0.92 & & & \\
\hline & Total & 767 & 3.49 & 0.72 & & & \\
\hline \multirow{7}{*}{ F4. Imagen afectiva } & 1. CEA & 188 & 3.26 & 1.04 & \multirow[t]{7}{*}{2.578} & \multirow[t]{7}{*}{0.025} & \multirow[t]{7}{*}{$6 / 3,5$} \\
\hline & 2. A & 88 & 3.26 & 0.87 & & & \\
\hline & 3. CBI & 158 & 3.46 & 0.71 & & & \\
\hline & 4. CSHU & 122 & 3.35 & 0.98 & & & \\
\hline & 5. CSA & 148 & 3.43 & 0.86 & & & \\
\hline & 6. CAP & 63 & 3.05 & 0.95 & & & \\
\hline & Total & 767 & 3.33 & 0.91 & & & \\
\hline \multirow{7}{*}{$\begin{array}{l}\text { Indicador global de } \\
\text { imagen organizacional } \\
\text { universitaria }\end{array}$} & 1. CEA & 188 & 3.43 & 0.55 & \multirow[t]{7}{*}{3.112} & \multirow[t]{7}{*}{0.004} & \multirow[t]{7}{*}{$2,6 / 3,5$} \\
\hline & 2. A & 88 & 3.31 & 0.54 & & & \\
\hline & 3. CBI & 158 & 3.55 & 0.50 & & & \\
\hline & 4. CSHU & 122 & 3.48 & 0.69 & & & \\
\hline & 5. CSA & 148 & 3.54 & 0.63 & & & \\
\hline & 6. CAP & 63 & 3.32 & 0.64 & & & \\
\hline & Total & 767 & 3.46 & 0.59 & & & \\
\hline
\end{tabular}

A: artes; CAP: ciencias agropecuarias; $\mathrm{CBI}$ : ciencias básicas e ingeniería; CEA: ciencias económico-administrativas; CSA: ciencias de la salud; CSHU: ciencias sociales y humanidades; DE: desviación estándar..

Fuente: elaboración propia basada en los resultados de la investigación.

la orientación y preparación universitaria $(\mathrm{F}=2.195 ; \mathrm{p}=0.050)$ es significativa y se percibe más alta en el Instituto de Ciencias de la Salud (media $=3.53$ ), muy probablemente porque se trata de un instituto en el que destaca la carrera de médico cirujano, que por su tradición genera egresados con un alto nivel académico.

La reputación de la institución $(\mathrm{F}=5.119 ; \mathrm{p}=0.000)$ es significativamente más alta en 4 institutos de mayor tradición y experiencia en la Universidad: el Instituto de Ciencias Básicas e Ingeniería (media =3.68), Instituto de Ciencias de la Salud (media =3.67), Instituto de Ciencias Sociales y Humanidades $($ media $=3.62)$ y el Instituto de Ciencias Económico Administrativas $($ media $=3.62)$. 
La madurez institucional $(\mathrm{F}=6.843 ; \mathrm{p}=0.000)$ es significativamente más alta en el Instituto de Ciencias Básicas e Ingeniería (media $=3.64)$.

La imagen afectiva $(\mathrm{F}=2.578 ; \mathrm{p}=0.025)$, es decir, el conjunto de emociones expresadas con respecto a la IES, se percibe significativamente más alta en el Instituto de Ciencias Básicas e Ingeniería (media $=3.46)$ y en el Instituto de Ciencias de la Salud (media $=3.43$ ).

En lo que respecta a la imagen global universitaria $(F=3.112 ; p=0.004)$ se identifica que es significativamente más alta (positiva) en el Instituto de Ciencias Básicas e Ingeniería (media = 3.55) y en el Instituto de Ciencias de la Salud (media = 3.54).

Tal como se muestra en la tabla 12 la percepción de la imagen organizacional universitaria se identifica significativamente más alta principalmente en 2 institutos: Ciencias Básicas e Ingeniería y Ciencias de la Salud. Estos resultados pueden obedecer a que ambos institutos cuentan con amplia trayectoria, excelente infraestructura, un destacado grupo de profesores de tiempo completo y programas educativos de excelencia académica, que en conjunto son factores que repercuten en que la percepción de la imagen universitaria sea más positiva.

\section{Conclusiones}

El objetivo de la presente investigación fue evaluar la imagen organizacional universitaria en una muestra de profesores y estudiantes de una IES. Para este propósito se efectuó la adaptación a población mexicana de las escalas de imagen organizacional de Beerli et al., 2002 y Russell y Pratt (1980). Los resultados obtenidos permiten confirmar la presencia de 4 factores (3 factores de imagen cognitiva y un factor de imagen afectiva) con adecuados niveles de validez y confiabilidad. Adicionalmente, se identificaron correlaciones significativas de los reactivos con su factor y con el total de la escala y correlaciones significativas entre los factores de la escala. Con estos resultados puede afirmarse que se cuenta con una escala que cumple con adecuados niveles de validez y confiabilidad para medir la imagen organizacional de IES y universidades.

También se identificaron diferencias estadísticamente significativas de la imagen organizacional percibida en cada una de las escuelas de la IES, lo que indica que cada una de ellas posee diferentes niveles en la imagen organizacional universitaria (cognitiva, afectiva y global), como resultado de la percepción que tanto estudiantes como profesores tienen de los aspectos académicos, administrativos y organizacionales de la IES evaluada.

En el futuro es recomendable evaluar la imagen organizacional en otras instituciones educativas de nivel bachillerato, licenciatura y posgrado a fin de retroalimentar a este tipo de organizaciones.

Si bien esta investigación se enfocó en evaluar la imagen organizacional universitaria de una IES, se recomienda que futuras investigaciones se enfoquen en la gestión de la imagen, proceso que debe basarse en la imagen construida por la organización y su repercusión en diversos públicos (empleados, alumnos, proveedores, gobierno y sociedad).

\section{Anexo 1.}

Escala de imagen organizacional universitaria. Basada en la adaptación de las escalas de Beerli et al., 2002 y Rusell y Pratt (1980) a población mexicana. 


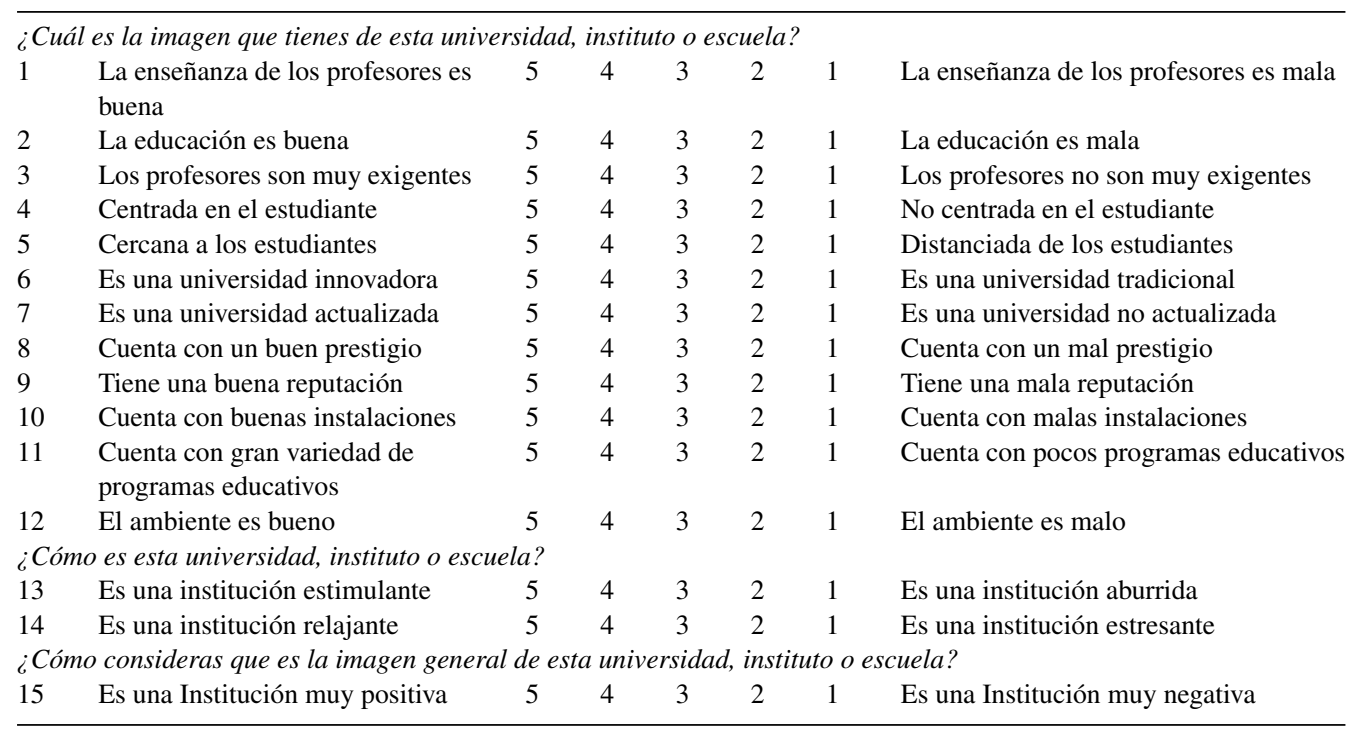

\section{Referencias}

Baker, S. y Brown, B. (2007). Images of excellence: Constructions of institutional prestige and reflections in the university choice process. British Journal of Sociology of Education, 28, 377-391. http://dx.doi.org/10.1080/01425690701253455

Beerli, A., Díaz, G. y Pérez, P. (2002). The configuration of the university image and its relationship with the satisfaction of students. Journal of Educational Administration, 40, 486-505. http://dx.doi.org/10.1108/09578230210440311

Berg, P. (1985). Organization change as a symbolic transformation process. En P. Frost, L. Moore, M. Meryl, C. Lundberg, y J. Marton (Eds.), Organizational culture. California: Sage.

Blázquez, M. y Peretti, M. (2012). Modelo para gestionar la sustentabilidad de las organizaciones a través de la rentabilidad, adaptabilidad e imagen. Estudios Gerenciales, 28, 40-50. http://dx.doi.org/10.1016/S0123-5923(12)70006-2

Brown, R. y Mazzarol, T. (2009). The importance of institutional image to student satisfaction and loyalty within higher education. Higher Education, 58, 81-95. http://dx.doi.org/10.1007/s10734-008-9183-8

Chun, R. (2005). Corporate reputation: Meaning and measurement. International Journal of Management Review, 7 , 91-109. http://dx.doi.org/10.1111/j.1468-2370.2005.00109.x

Dichter, E. (1985). What's in an image. The Journal of Consumer Marketing, 2, 75-79. http://dx.doi.org/10.1108/eb038824

Druteikiene, G. (2011). University image: Essence, meaning, theoretical and empirical investigation. Global Conference on Business and Finance Proceedings, 6, 167-174.

Dukerich, J., Golden, B. y Shortell, S. (2002). Beauty is in the eye of the beholder: The impact of organizational identification, identity, and image on the cooperative behaviors of physicians. Administrative Science Quarterly, 47, 507-533. http://dx.doi.org/10.2307/3094849

Duque, O. E. y Carvajal, P. L. (2015). La identidad organizacional y su influencia en la imagen: una reflexión teórica. Suma de Negocios, 6, 114-123. http://dx.doi.org/10.1016/j.sumneg.2015.08.011

Dutton, J., Dukerich, J. y Harquail, C. (1994). Organizational images and member identification. Administrative Science Quarterly, 39, 239-263. http://dx.doi.org/10.2307/2393235

Fombrun, C. y Van Riel, C. (2003). Fame \& fortune: How successful companies build winning reputations. New Jersey: Editorial Prentice Hall.

Galiniené, B., Marcinskas, A., Miskinis, A. y Druteikiene, G. (2009). The impact of study quality on the image of higher education institution. Informacijos Mokslal, 48, 68-81.

Giangrande, V. (1995). Em defesa do consumidor. Revista de Comunicação, 40, 20-21.

Gioia, D. y Thomas, J. (1996). Identity, image, and issue interpretation: sensemaking during strategic change in academia. Administrative Science Quarterly, 41, 370-403. http://dx.doi.org/10.2307/2393936 
Guédez, F. C. y Osta, T. K. M. (2012). Factores del a imagen institucional universitaria: perspectiva desde el sector del público interno, personal administrativo. Revista Ingeniería Industrial, 11, 71-84.

Guerra, V. y Arends, P. (2008). Medición de la imagen institucional de un postgrado universitario. Revista Ingeniería Industrial: Actualidad y Nuevas Tendencias, 1, 10-20.

Günalan, M. y Ceylan, A. (2014). The mediator role of organizational image on the relationship between jealousy and turnover intention: A study on health workers. The Journal of Social Sciences Institute, 17, 133-156.

Helgesen, O. y Nesset, E. (2007). Images, satisfaction and antecedents: Drivers of student loyalty? A case study of a Norwegian University College. Corporate Reputation Review, 10, 38-59. http://dx.doi.org/10.1057/palgrave.crr.1550037

Herrbach, O. y Mignonac, K. (2004). How organizational image affects employee attitudes. Human Resource Management Journal, 14, 76-88. http://dx.doi.org/10.1111/j.1748-8583.2004.tb00134.x

Huang, C. y Lien, H. (2012). An empirical analysis of the influences of corporate social responsibility on organizational performance of Taiwan's construction industry: using corporate image as a mediator. Construction Management and Economics, 30, 263-275. http://dx.doi.org/10.1080/01446193.2012.668620

Kotler, P. (1975). Marketing for non-profit organizations. New Jersey: Editorial Prentice-Hall.

Kotler, P. y Andreasen, A. (2008). Positioning the organization: Strategic marketing for nonprofit organizations. New Jersey: Editorial Prentice-Hall.

Mael, F. y Ashforth, B. (1992). Alumni and their alma mater. A partial test of the reformulated model of organizational identification. Journal of Organizational Behavior, 13, 103-123. http://dx.doi.org/10.1002/job.4030130202

Magierski, D. y Kassouf, N. (2003). Análise da imagen organizacional de universidades por meio da matriz familiaridadefavorabilidade. Revista de Administração Mackenzie, 4, 25-37.

McPherson, M. y Schapiro, M. (1998). The student aid game. New Jersey: Princeton University Press.

Nguyen, N. y Leblanc, G. (2001). Image and reputation of higher education institution in student's retentions decisions. The International Journal of Educational Management, 15, 303-311. http://dx.doi.org/10.1108/EUM0000000005909

Nolan, K. P. y Harold, C. M. (2010). Fit with what? The influence of multiple self-concept images on organizational attraction. Journal of Occupational and Organizational Psychology, 83, 645-662. http://dx.doi.org/10.1348/096317909X465452

Perozo, J. G. y Alcalá, S. M. (2008). Imagen corporativa de los Institutos Tecnológicos Universitarios de Maracaibo. Multiciencias, 8, 105-112.

Polat, S. (2011). The relationship between university students' academic achievement and perceived organizational image. Educational Sciences: Theory \& Practice, 11, 257-262, http://dx.doi.org/10.16 · Kocaeli University.

Russell, J. A. y Pratt, G. (1980). A description of the affective quality attributed to environments. Journal of Personality and Social Psychology, 38, 311-322. http://dx.doi.org/10.1037/0022-3514.38.2.311

Sartore-Baldwin, M.-L. y Walker, M. (2011). The process of organizational identity: What are the roles of social responsiveness, organizational image, and identification? Journal of Sport Management, 7, 489-505. http://dx.doi.org/10.1057/crr.2008.23

Stevens, R., McConkey, W., Cole, H. y Clow, K. (2008). College image: A strategy marketing dilemma. Services Marketing Quarterly, 29, 99-113. http://dx.doi.org/10.1080/15332960802126005

Torpor, B. (1983). Athletics and marketing. Marketing Higher Education, IX, 6.

Toto, M. F. y García, L. T. (2012). Vinculación, imagen y posicionamiento de una IES en la región de San Andrés Tuxtla, Veracruz. Ciencia Administrativa, 2, 11-24.

Traverso, C. J. (2005). Imagen interna de la institución universitaria. Modelo para el personal de administración y servicios. Revista de Economía y Empresa, XXIII(54 y 55), 95-112.

Treadwell, D. y Harrison, T. (1994). Conceptualizing and assessing organizational image: Model, images, commitment, and communication. Communication Monographs, 61, 63-85. http://dx.doi.org/10.1080/03637759409376323

Yim, Y. y Schafer, B. (2009). Police and their perceived image: How community influence officers' job satisfaction. Police Practice and Research, 10, 17-29. http://dx.doi.org/10.1080/15614260802128658 